\title{
Regulation of haemopoietic stem cells by OPN is mediated by specific interactions with $\alpha_{4} \beta_{1}$ and $\alpha_{9} \beta_{1}$ integrins
}

\author{
DN Haylock ${ }^{1}$, M Storan $^{1}$, J Grassinger ${ }^{1}$, B Williams $^{1}$, GA Whitty ${ }^{1}$, T Uede $^{1}$, SK Nilsson ${ }^{1}$ \\ ${ }^{1}$ Niche Laboratory, Australian Stem Cell Centre, Melbourne, Australia.
}

Osteopontin (Opn) is a phosphorylated glycoprotein, identified as an adhesive and migratory substrate for several cell types, which is highly expressed by osteoblasts within the hemopoietic stem cell (HSC) niche. We have previously demonstrated an unrecognised critical role for Opn in the regulation of the physical location and proliferation of HSC. Within the bone marrow the presence of Opn is restricted to the endosteal bone surface and we now have evidence that within this region, Opn exists in both the native as well as a cleaved form. In addition, we have shown that the cleaved form acts as a physiological negative regulator of HSC proliferation and differentiation through binding to both $\alpha_{4} \beta_{1}$ and $\alpha_{9} \beta_{1}$ integrins. We recently demonstrated for the first time the expression of $\alpha_{9} \beta_{1}$ on human HSC, with an inverse correlation between the expression of $\alpha_{9} \beta_{1}$ and CD38, demonstrating that this integrin is more abundantly expressed on more primitive HSC. In contrast $\alpha_{4} \beta_{1}$ expression mirrors that of CD38 and is expressed at higher levels on committed progenitors. Similarly, murine HSC also express $\alpha_{9} \beta_{1}$, with endosteal HSC exhibiting a higher level of expression compared to HSC from other bone marrow regions. Furthermore, we now have direct evidence of endogenous binding of Opn to both $\alpha_{4} \beta_{1}$ and $\alpha_{9} \beta_{1}$ on human cord blood HSC through coimmunoprecipitation experiments utilizing antibodies specific for $\alpha_{4}$ and $\alpha_{9} \beta_{1}$. In vitro, these cells specifically bind to OPN via both $\alpha_{4}$ and $\alpha_{9} \beta_{1}$. We have now explored the signalling pathways invoked following the interaction between OPN and these individual integrins expressed on HSC. Previous studies in other cellular systems have demonstrated that binding of ligands to $\alpha_{9}$ specifically signals via either SSAT or paxillin to result in de-adhesion or augmented cell migration respectively. We have recently made the novel observation that human cord blood CD34+ cells and murine endosteal HSC and central HSC cells express SSAT and paxillin. Thus despite the high degree of homology exhibited by the $\alpha_{4}$ and $\alpha_{9}$ cytoplasmic domains, binding of each integrin by one ligand can give rise to specific functional responses, attributed to the association of distinct adapter and signalling proteins. These data provide strong evidence that Opn is an important component of the HSC niche, participating in the attraction and retention of HSC to this region, where it acts as a negative regulator of HSC proliferation and differentiation via binding to one of two integrins and invoking specific signalling.

Cell Research (2008) 18:s10. doi: 10.1038/cr.2008.100; published online 4 August 2008

Correspondence: SK Nilsson

E-mail: susie.nilsson@stemcellcentre.edu.au

Susie K Nilsson, $\mathrm{PhD}$ is a Principal Investigator at the Australian Stem Cell Centre. Associate Professor Nilsson received a PhD in pathology from The University of Australia. She is currently an Associate Professor in Monash University and a Senior Research Fellow in the Department of Pathology, Melbourne University. She is on the editorial board and nominating committee of Experimental Hematology and has been chair and a member of the Emerging Leaders Task Force and a member of the Scientific Organizing Committee of the International Society of Experimental Hematology. Associate Professor Nilsson is both nationally and internationally recognized for her research interests devoted to understanding the hemopoietic stem cell niche and mechanisms that regulate haemopoietic stem cells. Please see her lab website for more details. http://www.stemcellcentre.edu.au/ 